\title{
Static Routing Algorithm of Nano-satellite Constellation
}

\author{
Yilong Shi ${ }^{1,2, a)}$, Xiujie Jiang ${ }^{1}$, Ping Zhu ${ }^{1,2}$, Yufeng Zhang ${ }^{1,2}$, Meng Liu ${ }^{1,2}$ \\ ${ }^{1}$ National Space Sciences Center, University of Chinese Academy of Sciences, Beijing, 100190, China. \\ ${ }^{2}$ University of Chinese Academy of Sciences, Beijing, 100190, China. \\ a) Corresponding author: shiyilong277@sina.com
}

\begin{abstract}
According to the work characteristics of Nano-satellite constellation, this paper studies the configuration of it and the static routing algorithm of it. Firstly, the coverage rates of different constellation configuration are given and the constellation configuration with the best coverage is confirmed. Secondly, the requirement of high-speed data transmission in Nano-satellite constellation is given. To solve the problem, the optimized static routing algorithm based on snap shot sequence algorithm is provided. Then, the coverage rates of different Nano-satellite constellation are simulated by sim tool kit. The high-speed data transmission model Nano-satellite constellation is established by using the NS3 network simulation platform. The results show that, $10 * 11$ classic Sun-synchronous orbit constellations has the best coverage rate of three latitude area. The performance of the designed static routing is better than traditional snap shot sequence algorithm in high-speed transmission scene.
\end{abstract}

Key words: Nano-satellite; coverage rate; constellation; static routing algorithm.

\section{INTRODUCTION}

With the development on distribution and miniaturization in satellite system, the Nano-satellite constellation become a developing trend. Several space demonstration tests and application studies have been implemented. Such as Flock-1 constellation [1], Space Technology 5 constellation plan [2]. Because of the resources constraints of Nanosatellite, it can't provide high-speed inter-satellite links as long as large satellites. And providing a large number of ground stations is not advisable. Therefore, it is hard to transmit a mass of data from constellation to the ground in time. To solve the problem, a new static routing strategy is designed. The strategy is constructing high-speed intersatellite link in polar regions to establish a high-speed network, the data is routed to the relay satellites which will pass through the ground station soon. Then the data on the relay satellites will download to the ground in a short time. The configuration of constellation is the foundation to the route design. Coverage rate is a usual performance index in constellation configuration. Existing satellite network routing algorithm is mainly aimed at large satellite networks. Wang et al [3] studied Cross -layer design of codes for satellite multimedia broadcast. Jiang et al [4] worked on multiclass traffic quality of service routing for low earth orbit (LEO) satellite networks. Agent-based load balancing routing for LEO satellite networks was researched by Rao et al [5]. Yao et al [6] analyzed satellite constellation of mid earth orbit (MEO) network. Wang et al [7] give the minimum number of snap in snap shot sequence routing. Wang [8] studied topological dynamics characterization for LEO satellite networks. Fischer et al [9] give a routing algorithm for spacecraft networks. Routing in delay tolerant networks is analyzed by Cao [10]. The energy efficiency of satellite networking is studied by Alagoz [11]. This paper analyzes configuration of the Nano-satellite constellation, studies the static routing suitable for high speed digital transmission. 


\section{CONSTELLATION AND STATIC ROUTING ANALYSIS}

\section{Nano-Satellite Constellation Analysis}

The factors to consider in the design of Nano- satellite constellation include the configuration of the networks satellite, the number of the orbit, and the number of satellites in each orbital plane. When the total number of satellites are fixed, the number of orbital planes and the number of satellites in each orbit are inversely related.

To analysis of Nano-satellite constellation coverage objectively, should divide the earth into low latitudes region $\left(-30^{\circ} \sim 30^{\circ}\right)$, middle latitude region $\left(-30^{\circ} \sim-70^{\circ} \cup 30^{\circ} \sim 70^{\circ}\right)$, and the high latitude region, $\left(-70^{\circ} \sim-90^{\circ} \cup 70^{\circ} \sim 90^{\circ}\right)$. Since most of the world's population lives in the middle and lower latitudes, global uniform coverage takes precedence. Economic factor is another factor should be taken into consideration. As the number of satellite orbit increases, so does the cost of launch.

That means all factors need to be taken into account. Suppose that the height of the orbit is $600 \mathrm{~km}$. Take the classic homogeneous constellations as an example to analysis the coverage area in orbit inclination angle. Simulation of coverage area of different orbit inclination angle is done by Sim Tool Kit (STK). The simulation results are showed below.

TABLE 1. Coverage rate list of different inclination

\begin{tabular}{cccc}
\hline $\begin{array}{c}\text { Orbit inclination angle } \\
\left({ }^{\circ}\right)\end{array}$ & $\begin{array}{c}\text { Low latitude coverage rate } \\
(\%)\end{array}$ & $\begin{array}{c}\text { Mid latitude coverage rate } \\
(\%)\end{array}$ & $\begin{array}{c}\text { High latitude coverage rate } \\
(\%)\end{array}$ \\
\hline $97.79(\mathrm{SSO})$ & 86.00 & 97.30 & 90.20 \\
90 & 76.60 & 88.80 & 85.60 \\
80 & 85.20 & 97.10 & 90.70 \\
70 & 87.60 & 96.90 & 67.20 \\
60 & 87.00 & 95.40 & 23.60 \\
50 & 90.80 & 86.60 & 0 \\
40 & 96.30 & 61.90 & 0 \\
30 & 98.60 & 39.00 & 0 \\
\hline
\end{tabular}

High inclination orbits have contributed to the coverage of low, medium and high latitudes. The sun synchronous orbit (SSO) has a more balanced coverage rate in low, medium and high latitudes. As shown in the table, the sun synchronous orbit has $86 \%$ low latitude coverage rate, $97.30 \%$ mid latitude coverage rate, $90.20 \%$ high latitude coverage rate. The coverage figure of SSO is shown in the figure 1.

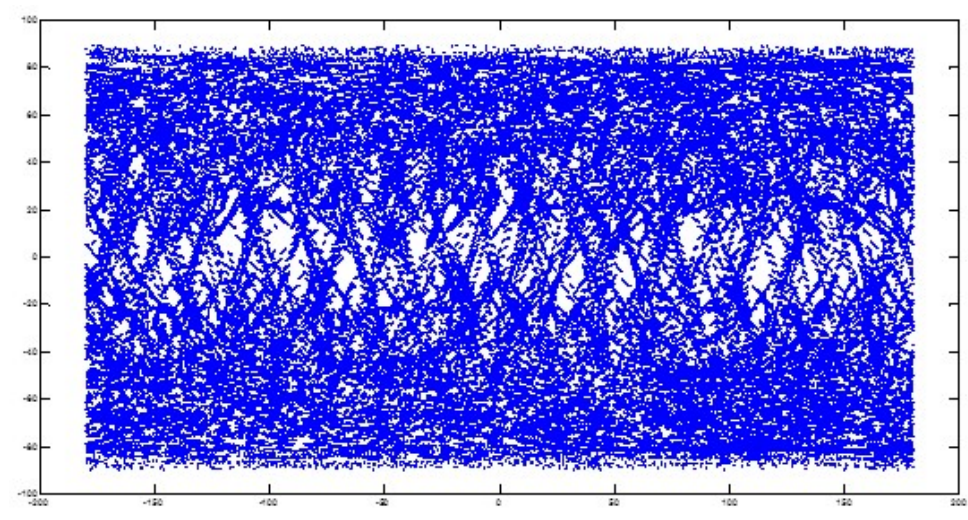

FIGURE 1. The coverage of sun synchronous orbit

Table 1 and Figure 1 show that SSO is more balanced in low, medium and high latitudes than the other orbit inclination angle. In order to achieve balanced coverage in medium, low, high latitude, the SSO orbital should be adopted. In the condition of fixed number of network satellites, the coverage of the lower latitudes will tend to be 
better as the orbital number increases. Taking 110 satellite constellations as an example, studies the effective on coverage by different orbit. Simulate result is shown below.

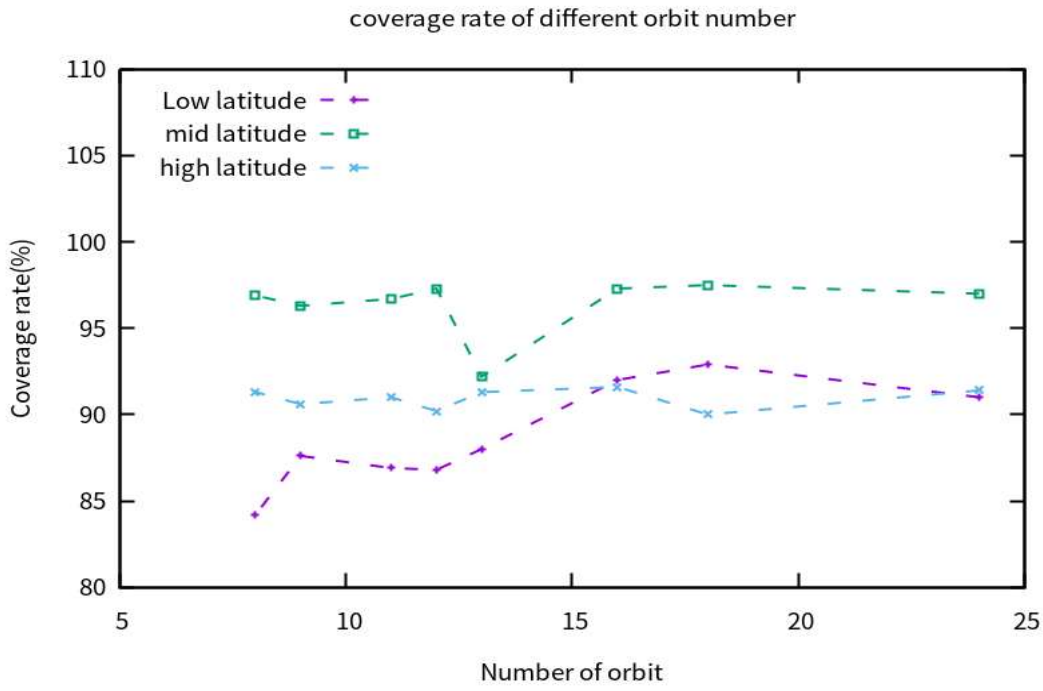

FIGURE 2. The coverage of different number of orbit

Figure 2 shows that the coverage rate of all latitudes will be better when the orbital increases. However, as the number of satellite orbit increases, so does the cost of launch. Synthesizes all the factors, $10^{*} 11$ classic SSO constellations is the best configuration.

\section{Static Routing Protocol for Constellation.}

The study on static routing is based on the SSO constellation. Because of the resources constraints of Nanosatellite, it is hard to provide high-speed inter-satellite links as long as large satellites. Transmitting data from constellation with a large number of satellites to the ground in time will be a problem. Therefore, a static routing algorithm should be designed. Traditional snap shot sequence(SSS) routing algorithm is not suitable for the scene. Then an optimized routing algorithm based on SSS is given. The basic idea of the SSS algorithm is to divide the satellite system period into small time slices. The minimum number of snapshots in a SSO orbit period is:

$$
\left\{\begin{array}{l}
n_{\text {snap }, \min }=\frac{2 N}{P}, \text { when } \frac{N}{P} \in 2 N \\
n_{\text {snap }, \min }=\frac{4 N}{P}, \text { when } \frac{N}{P} \in 2 N+1
\end{array}\right.
$$

where $n_{\text {snap,min }}$ is minimum number of snapshots in a SSO orbit period, $N$ is the total number of satellites in the constellation and $P$ is the number of orbital plane.

Suppose the length of each route record is $L_{r t}$ item bytes, the size of routing table on satellite is:

$$
M_{\text {snap }}=(N-1) L_{r t_{-} \text {item }}
$$

where $M_{\text {snap }}$ is routing table size of satellite in each time slice. In an orbit period, the overhead of updating the routing on a SSO satellite is: 


$$
S_{S S S, I S L}=\sum_{i=1}^{n_{\text {snap }}} \sum_{j=1}^{N} M_{i, j} L_{i, j}
$$

where $M_{i, j}$ is the size of the routing table of the jth satellite which was updated in the ith snapshot and $L_{i, j}$ is the number of hop from the jth satellite to the ground station in the ith snapshot.

If all the satellite routing tables are updated in each snapshot, the overhead of updating the routing table in an orbit period is:

$$
S_{S S S, I S L} \approx n_{\text {snap }} \bar{L}(N-1) L_{r t_{-} \text {item }} \approx n_{\text {snap }} \frac{\left(\left[\frac{N}{2 P}\right]+P-1\right)}{2}(N-1) L_{r t_{-} \text {item }}
$$

where $\bar{L}$ is the average hop between the source and the destination satellites of the SSO constellation the memory capacity of satellite in an orbit period is:

$$
M_{C}=n_{\text {snap }} M_{\text {snap }}=n_{\text {snap }}(N-1) L_{r t_{-}} \text {item }
$$

where $n_{\text {snap }}$ is the number of snapshots in an orbit period, For Nano-satellite, maintaining high-speed intersatellite links throughout the whole period is hard. The traditional SSS routing is not suitable for the scene in which data from the Nano-satellite constellation needs to transmit to the ground station in time.

The way to solve the problem is to design an optimized static routing algorithm based on SSS routing. It is feasible for Nano-satellite to maintain high-speed inter-satellite link when the satellites are in the polar region. The data can be routed to the relay satellite that is about to go through the ground station. The routing strategy can reduce the number of node in high-speed network to the orbit numbers (each orbital plane has a relay node). When optimized algorithm is used, $\mathrm{N}$ is equal to $\mathrm{P}$. The minimal overhead in an orbit period comes to:

$$
S_{S S S, I S L} \approx \frac{T}{\square t} \times \frac{(P-1)^{2}}{2} L_{r t_{-}{ }^{i t e m}}
$$

Where $T$ is the orbit period, $\Delta t$ is the length of the time slice.

\section{RESULTS AND DISCUSSION}

Suppose $L_{r t \text { item }}$ is 2 bytes, the number of satellite in each orbit is fixed to $11, T$ is 3 hours, $\square t$ is 30 seconds. When key performance index is simulated by NS3, the overhead of updating routing table in an orbit period is shown below: 


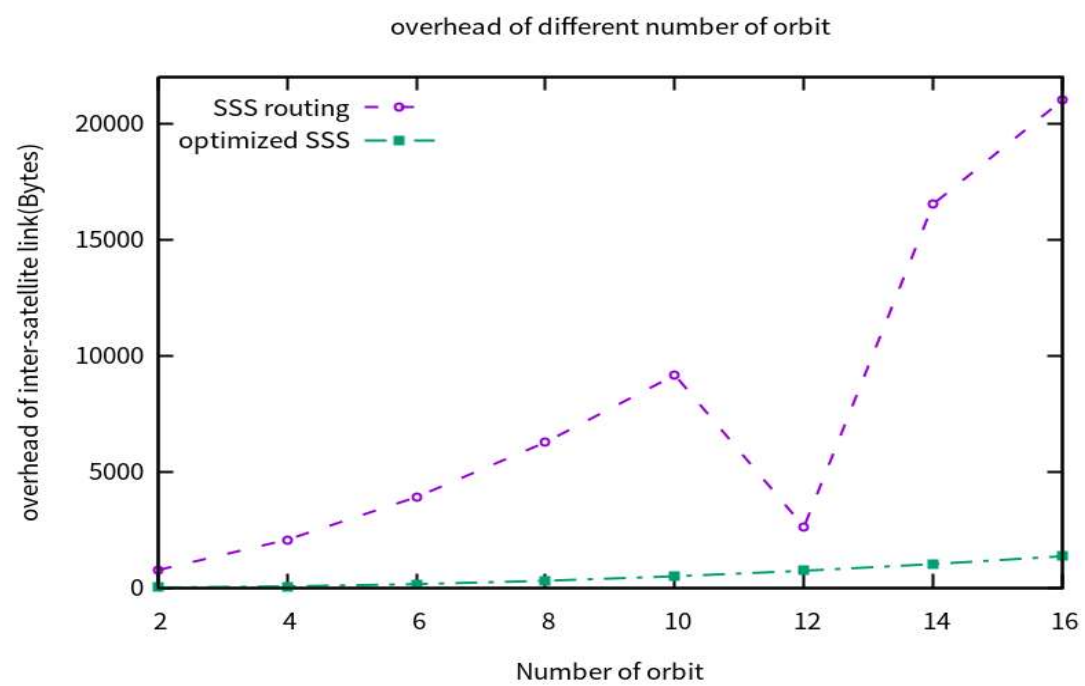

FIGURE 3. Overhead of different orbit number

Figure 3 is a diagram of the overhead of optimized SSS and SSS algorithm in different number of orbit. When the number of orbit comes to 16, the improvement of overhead by using optimized SSS reaches max. While orbit number comes to 12, the improvement of overhead reaches minimum. The simulation results show that the average overhead of the network is improved when the node adopts the optimized SSS protocol.

Suppose the routing table upload only once throughout the whole orbit period, the configuration of constellation is $10^{*} 11$. The storage occupancy in an orbit period when different time slice is chosen is shown in the figure below:

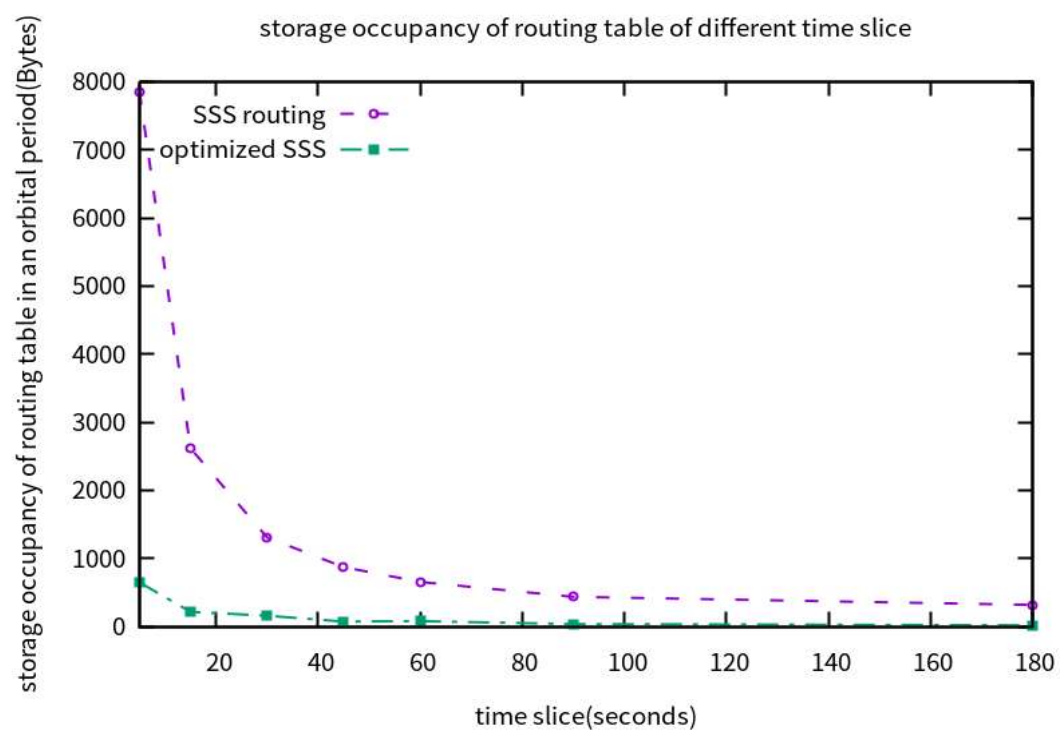

FIGURE 4. The storage occupancy of the two protocol in different time slice

In Fig 4, simulation results show that storage occupancy of optimized SSS has been reduced significantly when different time slice is chosen. It means that the optimized SSS has a better performance on both overhead and storage occupancy than SSS algorithm. 


\section{CONCLUSION}

This paper analyzes the Nano-satellite constellation configuration and the static routing algorithm of it. It focuses on the Nano-satellite constellation with the best coverage rate and the optimized SSS routing protocol to meet the requirement of high-speed data transmission. The coverage rate of constellation is simulated by STK and the key performance index of static routing protocol is simulated by NS3. According to the simulation result by STK, 10*11 classic SSO constellations has the best coverage rate of three latitude area. The simulation results from NS3 show that the optimized SSS routing algorithm has a better performance on routing overhead and storage occupancy than traditional one. It means that the study on static routing protocol is meaningful to solve the problem that large volumes of data in the constellation need to transmit to the ground station in time.

\section{REFERENCES}

1. Z. B. WANG, Z. Y. WANG, X. M. G, Q. GUAN: Cross -layer design of LT codes and LDPC codes for satellite multimedia broadcast/multicast services (Chinese Journal of Aeronautics, Beijing, 2013), pp. 1269 -1275.

2. Y. Tomioka, K. Yoshida, Y. Sakamoto, T. Kuwahara: Lessons Learned on Structural Design of 50kg Microsatellites. Based on Three Real-life Micro-satellite Projects. (2012 IEEE/SICE International Symposium on System Integration, Fukuoka-shi,2012), pp. 319-324.

3. S. Bandyopadhyay, G. Subramanian, R. Foust, D. Morgan, S. Chungl: A Review of Impending Small Satellite Formation Flying Missions. (In Proceedings of the 53rd AIAA Aerospace Sciences Meeting, Kissimmee FL, 2015), pp. 1611-1623.

4. H. Kang: Research on the MAC Protocol of Wireless Network for the Fractionated Payload. Ph.D. thesis, The University of Chinese Academy of Sciences. 2016.

5. S. Lan, Q. Chen, J. Zhang, X. Zhang, J. Li: A taxonomy of energy efficiency strategies for cube sat cluster formation networks. (Proceeding of the 64th International Astronautical Congress (IAC) Beijing: International Astronautical Federation, Beijing, 2013), pp. 11486-11493.

6. X. Yi, Z. Sun, F. Yao, Y. Miao: Satellite constellation of MEO and IGSO network routing with dynamic grouping. (International journal of Satellite Communications and Networking, Chichester,2013), pp. $277-302$.

7. J. Wang, L. Li, M. Zhou: Topological dynamics characterization for LEO satellite networks. (Computer Networks, Amsterdam, 2007), pp. 43-53.

8. D. Fischer, D. Basin, K. Eckstein, T. Engel: Predictable Mobile Routing for Spacecraft Networks. (IEEE Transactions on Mobile Computing, Los Alamitos, 2013), pp. 1174 1187.

9. M. Bansal, J. Mehlman, S. Katti, P. Levis : Open Radio: A Programmable Wireless Data plane (Workshop on Hot Topics in Software Defined Networks, Helsinki, 2012), pp. 109 114.

10. Y. CAO, Z. SUN: Routing in Delay/Disruption Tolerant Networks: A Taxonomy, Survey and Challenges. (Communications Surveys \& Tutorials IEEE, Piscataway, 2013), pp. 654 677.

11. F. AlAGOZ, R. G. Gu: Energy Efficiency and Satellite Networking: A Holistic Overview. (Proceedings of the IEEE, Piscataway, 2011), pp. 1954 1979. 